\title{
INVESTIGATIONS ON THE GEOMETRIC QUALITY OF CAMERAS FOR UAV APPLICATIONS USING THE HIGH PRECISION UAV TEST FIELD ZOLLERN COLLIERY
}

\author{
H.-J. Przybilla ${ }^{\text {a, }}$ *, M. Gerke ${ }^{\text {b }}$, I. Dikhoff ${ }^{\text {b }}$, Y. Ghassoun ${ }^{\text {b }}$ \\ ${ }^{\text {a }}$ Bochum University of Applied Sciences, Lab of Photogrammetry, D-44801 Bochum, Germany - \\ Heinz-Juergen.Przybilla@hs-bochum.de \\ ${ }^{\mathrm{b}}$ Institute of Geodesy and Photogrammetry, TU Braunschweig, Germany, Bienroder Weg 81, 38106 Braunschweig, Germany \\ \{m.gerke, i.dikhoff, y.ghassoun\}@tu-bs.de
}

Commission ICGW I/II

KEY WORDS: Unmanned aerial vehicle, UAV test field, camera sensors, geometric quality parameters, workflow

\begin{abstract}
:
The geodetic-photogrammetric test field at the industrial monument Zollern colliery in Dortmund offers a scenario for carrying out geometric and radiometric tests of UAV systems. The foundation for this builds a geodetic precision network (position and height accuracy approx. $2 \mathrm{~mm}$ ) with a total of 45 ground control points, distributed over an area of approx. 7 hectares. Within the scope of a campaign carried out in autumn 2017, various UAV sensor systems were tested under comparable conditions. Within this paper geometric investigations of two current DJI cameras, Zenmuse X4S (20 Mpix) and X5S (20.8 Mpix), as well as a Phase One IXU 1000 (100 Mpix) are presented. While the Zenmuse cameras reflect the current state of development of the manufacturer DJI, the medium format camera system from Phase One is primarily settled in the classic aerial segment. However, the desire for increased measurement accuracy (e. g. for engineering applications) also makes such a high-performance sensor interesting for UAV applications.

In addition to the configuration of the test field, the system comparison requires identical parameters for flight planning, in particular image overlapping, a complete cross flight configuration at different flight altitudes and the definition of a uniform ground resolution $(\mathrm{GSD}=14 \mathrm{~mm})$.
\end{abstract}

The investigations show clear differences in the achievable quality of the cameras. Though the high-priced Phase One system shows the best results, the most cost-effective system, the Zenmuse X4S, delivers only slightly worse results. In contrast, the Zenmuse X5S performs significantly worse than the other systems, mainly resulting from the mechanically unstable camera concept with interchangeable lenses. Finally, the comparison of the software products Pix4D Pix4Dmapper, Inpho UASMaster by Trimble and Agisoft PhotoScan partly shows significant differences in the results of image orientation. In particular in settings with sparse GCP usage the results vary considerably, indicating different strategies on how the residuals are distributed and the datum is defined, mostly Pix4Dmapper outperforms the others. In better GCP configurations there is no significant difference between Pix4mapper and Agisoft PhotoScan, while UASMaster does never deliver the best results.

\section{INTRODUCTION}

UAV-based imaging has become an established geodeticphotogrammetric data acquisition method, which is used in many application areas with various demands to the quality of the results - from decimeter to millimeter scale- and due to its great flexibility. In the context of a system procurement, users are usually interested in highly developed UAV platforms, often neglecting to inform themselves sufficiently about the quality of the integrated camera. The selection and quality/geometry of the camera is the most important factor with regard to the results to be achieved (e.g. 3D point clouds, orthoimages, etc.). The market for digital cameras, which are used in UAV-based scenarios, is subject to continuous change, with new cameras being launched very frequently, so that existing systems become obsolete quickly.

UAV of the manufacturer DJI have a considerable market presence and are primarily used in film and video productions. In the meantime, however, they have also found widespread use in geodetic applications. The Zenmuse X4S (20 Mpix) and X5S (20.8 Mpix) DJI cameras reflect the current state of development of the manufacturer but come with very different designs (Table 1, Figure 1).

While the proprietary DJI cameras are used in conjunction with the Phantom 4 Pro and Inspire 2 series, the Phase One IXU 1000 (100 Mpix) is a medium format camera system that is typically used on classic aerial photography platforms. For the Zollern colliery tests, a powerful UAV from Coptersystem (Coptersystems 2019), with a maximum take-off weight of $10 \mathrm{~kg}$, was used. The achievable flight time with this copter is approx. 20 minutes and thus comparable to that of a DJI flight platform (Figure 1).

In manned aerial photogrammetry, the establishment and use of test fields has been a field-proven procedure for investigating the quality of photogrammetric camera systems over many decades. In this context, the Vaihingen/Enz test field of the University of Stuttgart (Cramer \& Krauß 2008) displays a prominent example. Furthermore, test fields are also used for the in-situ calibration of digital aerial cameras (Mueller \& Neumann 2016). However, these test fields do not meet the requirements for testing UAV systems. In addition to the size (adapted to the classical image flight), the accuracy of the reference points, which in the case of

\footnotetext{
* Corresponding author
} 
a UAV test field must be in the sub-centimeter range (chapter 2), is primarily lacking. district of Bövinghausen in the art Nouveau style. After its closedown at the end of the 1960s, the Regional Association Westphalia Lippe (LWL) integrated the colliery into the

\begin{tabular}{|c|c|c|c|}
\hline Manufacturer & Phase One / Coptersystems & \multicolumn{2}{|c|}{ DJI } \\
\hline Camera & Phase One IXU1000 & Zenmuse X5S & $\begin{array}{c}\text { Zenmuse X4S } \\
\text { Phantom 4 Pro) }\end{array}$ \\
\hline Lens & $\begin{array}{c}\text { Rodenstock } \\
50 / 5.6\end{array}$ & $\begin{array}{c}\text { DJI MFT ASPH } \\
15 / 1.7\end{array}$ & No \\
\hline Interchangeable Lens & $\begin{array}{c}\text { Yes (mechanically stabi- } \\
\text { lized) }\end{array}$ & Yes \\
\hline Focus & Mechanical: $\infty$ & Electronical: $\infty$ & Electronical: $\infty$ \\
\hline Shutter & Central (mechanical) & Rolling & Central (mechanical) \\
\hline Resolution [MPx] & 100 & 20,8 & $13.2 \times 8.8$ \\
\hline Sensor Format [mm] & $53.4 \times 40.0$ & $5.280 \times 3956$ & $5.472 \times 3648$ \\
\hline Number of Pixel & $11.608 \times 8708$ & 3,28 & 2,4 \\
\hline Pixelsize [ $\mu \mathrm{m}]$ & 4,6 & 15 & 83,8 \\
\hline Focal Length [mm] & 50 & $73,6^{\circ}$ & 800 \\
\hline $\begin{array}{c}\text { Field of View (FOV) / (diag- } \\
\text { onally) }\end{array}$ & $67,4^{\circ}$ & 2.200 & \\
\hline Price [€] & $50-60.000\left(^{*}\right)$ & & \\
\hline
\end{tabular}

(*) depending on the lens used

Table 1. Technical data of the camera systems under test

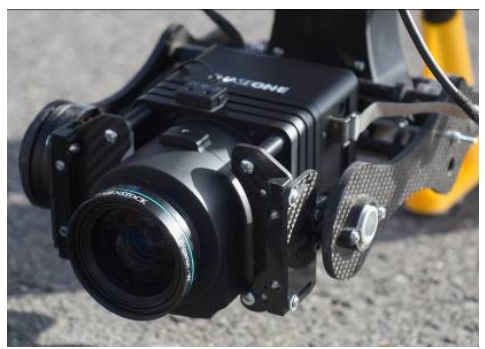

Phase One IXU 1000 (100 Mpix)

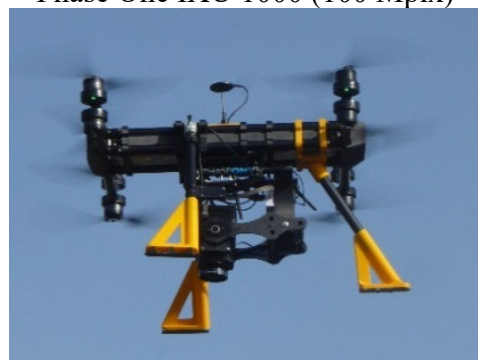

Coptersystems Multicopter

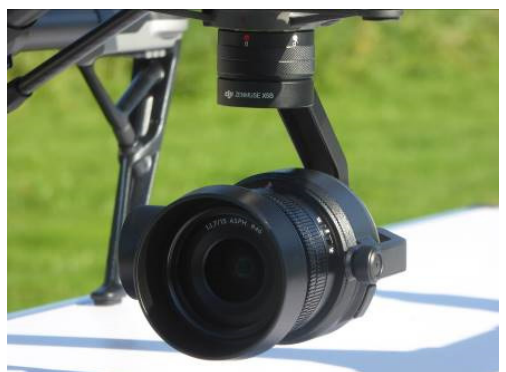

DJI Zenmuse X5S (20,8 Mpix)

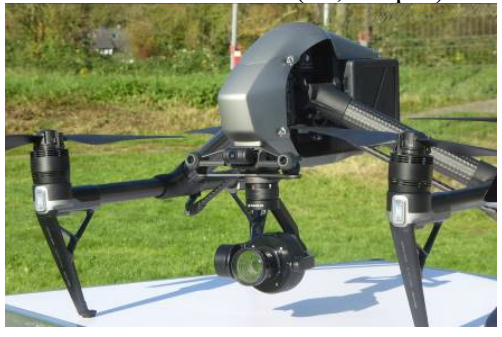

DJI Inspire 2

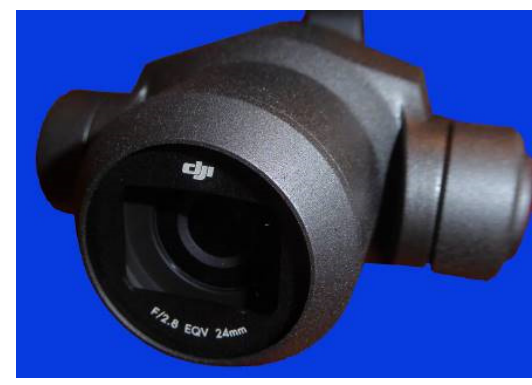

DJI Zenmuse X4S (20 Mpix)

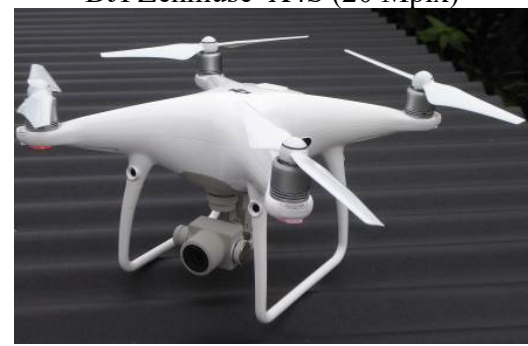

DJI Phantom 4 Pro

Figure 1. Cameras and carrier platforms under test

The aim of this study is to compare the geometric quality of the cameras involved. Additionally, the investigations focus on parameters that can influence the geometric quality of the imageblocks.

\section{THE UAV TEST FIELD ZOLLERN COLLIERY}

The UAV test field at the industrial museum Zollern colliery in Dortmund (LWL 2019) was established by the Bochum University of Applied Sciences (HSBO) in 2014 and has been the foundation for various campaigns so far (Nex et al. 2015; Przybilla et al. 2015; Gerke \& Przybilla 2016; Cramer et al. 2017; Przybilla et al. 2017). Further examples concerning experiences with UAV test field calibration are e.g. presented by Oniga et. al (2018). The Zollern colliery was built in 1898-1904 on the western outskirts of Dortmund $\left(51^{\circ} 31^{\prime} 4^{\prime \prime} \mathrm{N}, 7^{\circ} 20^{\prime} 5^{\prime \prime} \mathrm{O}\right)$ in the decentralized Westphalia industrial museum in 1981 (Wikipedia 2019). Today, the colliery is not only a museum with permanent and changing exhibitions, but also a place for local recreation as well as cultural and scientific events.

The UAV test field set up by the HSBO covers almost the entire area of the colliery. Its extension is $320 \mathrm{~m} \times 220 \mathrm{~m}$ (approx. $7 \mathrm{ha}$ ). The highest vertical objects are given by two pitheads with about $40 \mathrm{~m}$ height. In general, the test field is based on 45 signalized ground control points arranged in a grid (Figure 2). An overview of the implemented geodetic measurements for establishing the UAV test field is given in Figure 3, including the quality of the results (Przybilla et al. 2018). 


\section{DATA CAPTURING CONCEPT}

In order to ensure comparability of the camera systems within the test, a uniform ground resolution (GSD) of $14 \mathrm{~mm}$ was defined in advance for all systems (flight configuration: Regular / R).
Consequently, the platforms were operated at different flight altitudes (Table 2). Additionally, this was supplemented by flights in a cross arrangement (Cross / C), whereby the flight altitude deviated from the normal arrangements by $20 \%$ in each case (Figure 4). It should be noted that the extension of the
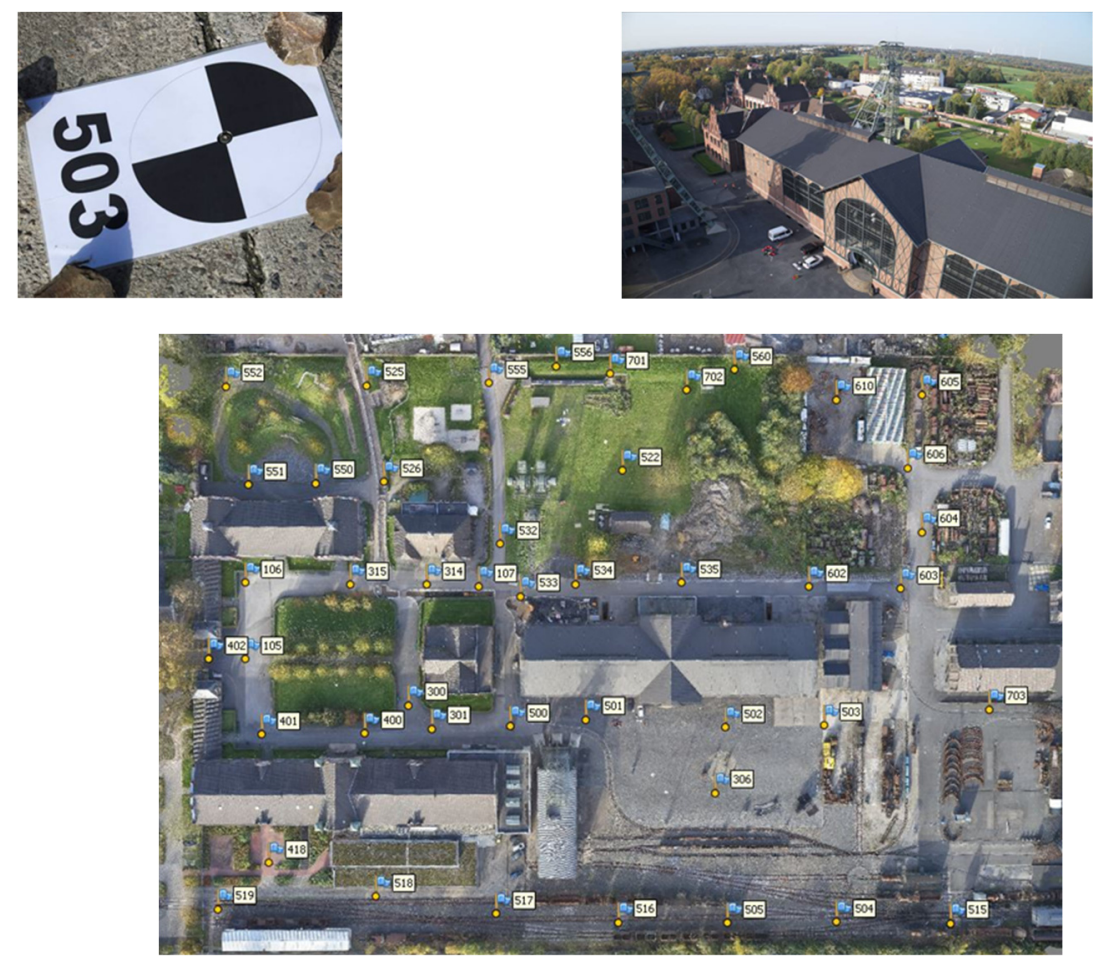

Figure 2. UAV test field Zollern colliery. Top: Target for signalizing the GCPs and the view of the area. Bottom: Point cloud with signalized GCPs (UAV flight from October 2017)

\begin{tabular}{|c|}
\hline RTK-GNSS \\
(SAPOS-HEPS) \\
\hline $\begin{array}{c}\text { Double measurement } \\
30 \text { epochs each }\end{array}$ \\
UTM coordinates, \\
ellipsoidal heights and \\
NHN heights \\
\hline \\
\hline Reference points for \\
30 net adjustment \\
\hline
\end{tabular}

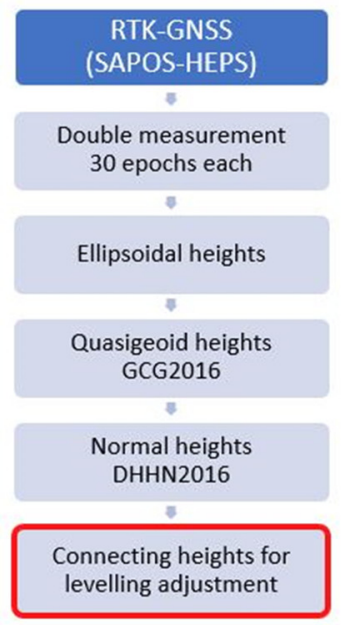

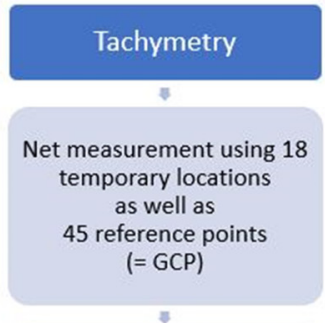

Net adjustment in a local north-oriented tangential coordinate system.

Zero point in the middle of the measurement area

\section{Back transformation of the adusted coordinates from the local coordinate system into the \\ ETRS89 coordinate system}

$$
\begin{gathered}
\text { Tachymeter positions: } \\
\sigma_{0}=1 \mathrm{~mm} \\
\text { Control points / } \\
\text { (Reference points): } \\
\sigma_{0}=1-4 \mathrm{~mm}
\end{gathered}
$$

Figure 3. Workflow of geodetic measurements and data processing (GCG2016 - German Combined QuasiGeoid, the height reference for the transition between geometric heights in ETRS89/DREF91 and physical heights in DHHN2016, the German height reference system). 
recording concept by the cross arrangement was used because of its positive effect on the in-situ calibration of the camera, as shown before (Przybilla et al. 2015; Gerke \& Przybilla 2016).

\begin{tabular}{|c|c|c|c|}
\hline & $\begin{array}{c}\text { Phase One } \\
\text { IXU1000 }\end{array}$ & $\begin{array}{c}\text { DJI Zenmuse } \\
\text { X5S }\end{array}$ & $\begin{array}{c}\text { DJI Zenmuse } \\
\text { X4S }\end{array}$ \\
\hline $\begin{array}{c}\text { Regular } \\
(\mathrm{R})\end{array}$ & $120 \mathrm{~m}$ & $60 \mathrm{~m}$ & $50 \mathrm{~m}$ \\
\hline Cross (C) & $148 \mathrm{~m}$ & $72 \mathrm{~m}$ & $60 \mathrm{~m}$ \\
\hline
\end{tabular}

Table 2. Systems, flight arrangement and altitude

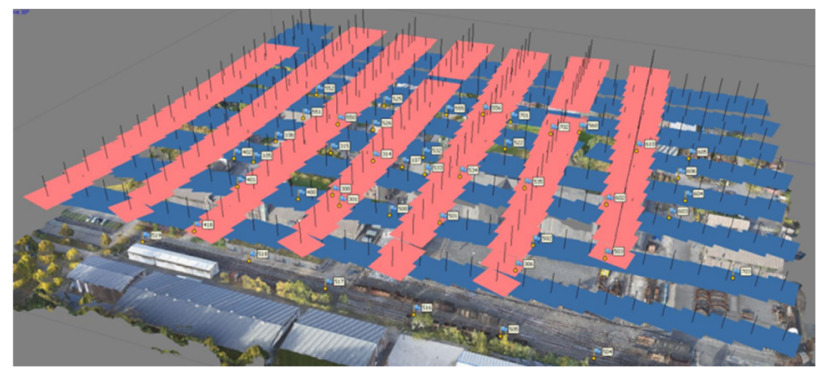

Figure 4. Flight arrangement Regular (R) and Cross (C), (Image block with Zenmuse X4S)

\section{RESULTS OF INVESTIGATION}

One of the investigation's focus was the determination of parameters that influence the geometric quality of the image blocks (Figure 5). Therefore, the aim was to investigate the influence of those parameters. To do so, the root mean square calculations with Agisoft PhotoScan (focal length: f; principle point: cx, cy; radial symmetric distortion: $\mathrm{k} 1-\mathrm{k} 3$; affinity and non-orthogonality: b1, b2; tangential asymmetric distortion: $\mathrm{p} 1$, $\mathrm{p} 2)$.

\subsection{Effects of ground control point configuration}

The number and arrangement of the control points usually has a significant influence on the block geometry. Substantial effects of different ground control point configurations on the final products (e. g. DEM) were shown by Lindstaedt \& Kersten (2018) in several UAV-based surveys in Ethiopia. On the one hand, a high number of control points stabilizes the block, while on the other hand, it requires a considerable effort for terrestrial surveying on site. Since UAV systems actually provide differential GNSS (RTK) for block referencing only to a small extent (Grayson et al. 2018), the distribution of the control points is often based on schemes from the time of the "analogue" image flight (Figure 6). Deviating from the distribution shown here, in practice, all points are measured as 3D coordinates - often using GNSS.

Figures 7-9 show the effects of the varying ground control point distributions for the three cameras tested within this investigation. The results are based on a block reference with 45 , 22, 12 and 5 GCP respectively. Essentially, those GCPs are distributed in a grid over the area (Figure 2). The reduction of the GCP leads in turn to an increase of the number of check points (CP), from 0 to 23,33 up to $40 \mathrm{CP}$, thus offering the possibility to verify the block geometry. The basis for all calculations is a cross flight (RC), which consists of two separate partial flights. The image data format used in the following is an uncompressed

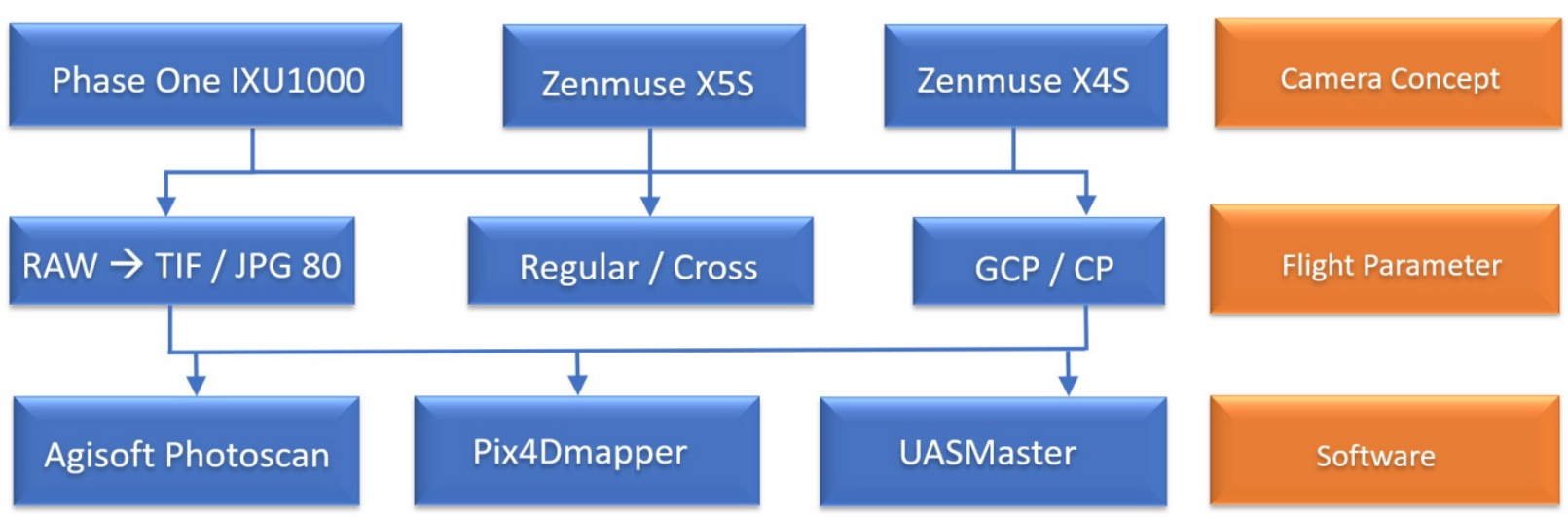

Figure 5. Parameters influencing the geometric quality of the image blocks

errors (RMSE values) at the check points (CP) were evaluated. These statistical values are results of the bundle block adjustments (BBA) using the evaluation software Agisoft PhotoScan, Pix4Dmapper and Inpho UASMaster respectively. The calculation variants are depending on the following parameters:

- block configuration (number of control points and arrangement),

- number of cameras in the self-calibration process (interior orientation),

- $\quad$ flight arrangement (Regular $-\mathrm{R} /$ Cross $-\mathrm{C}$ ),

- evaluation software used.

Importantly, the set of interior orientation parameters to be estimated for each camera tested, was identical for all subsequent
TIF, which was derived from the recorded RAW image data with the Capture One software from Phase One. For the results shown in Figure 7, ONE set of calculated interior orientation parameter is introduced (UNIFIED - identical for both partial flights).

The comparison of the RMSE values at the check points (at which any existing model bending can be detected) shows significant differences between the different systems. The largeformat Phase One camera system provides the lowest RMSE values - a result that can be confirmed for all control point arrangements.Even in the minimum arrangement (5 GCP - in the block corners and the block center), this leads to $3 \mathrm{D}$ accuracies that are well below the GSD of $14 \mathrm{~mm}$. Both, the large image format and the mechanical stability of the camera, are responsible for these results. 


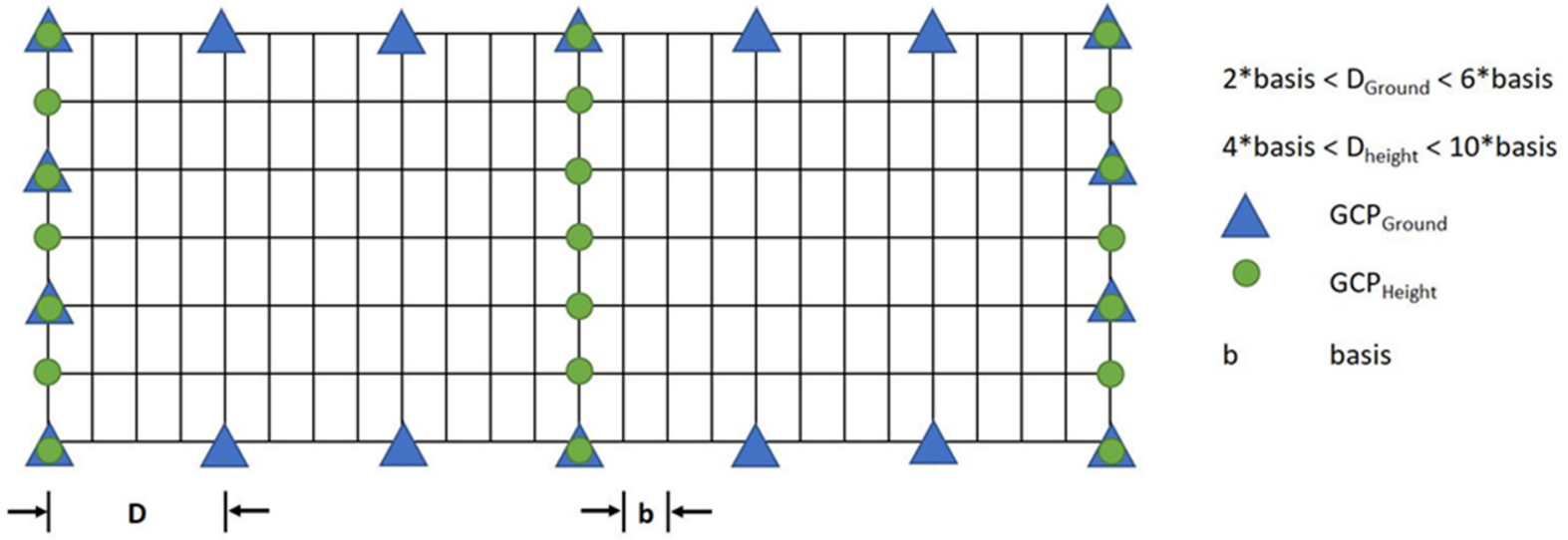

Figure 6. Schematic arrangement of GCPs during (analogue) image flight (Kraus 1994)

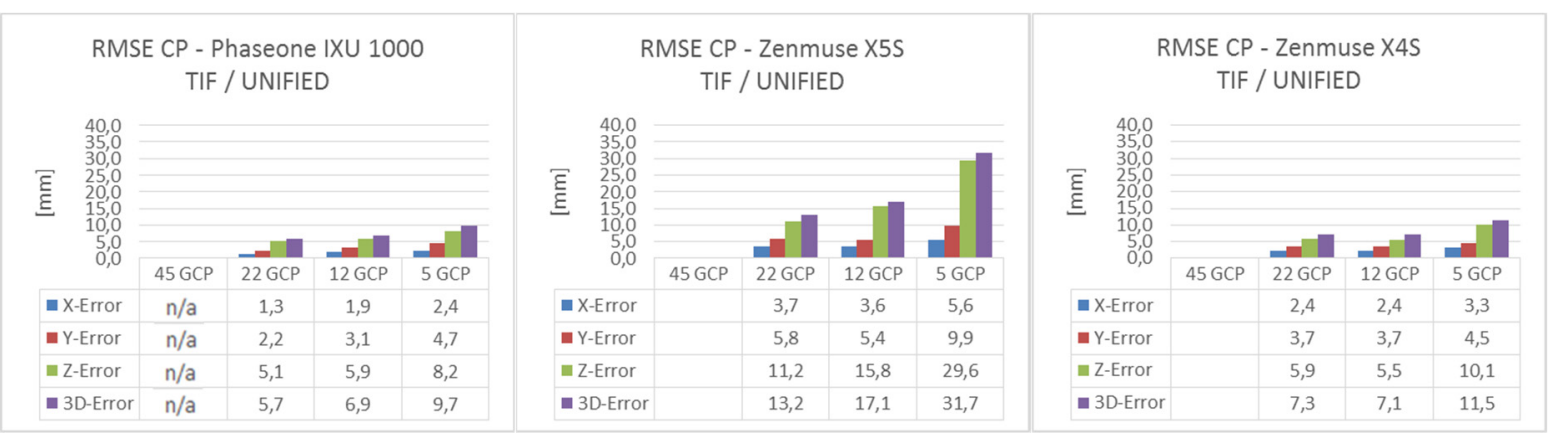

Figure 7. RMSE values at CPs. Flight arrangement: RC. ONE set of interior orientation parameters (UNIFIED). Calculation: Agisoft PhotoScan

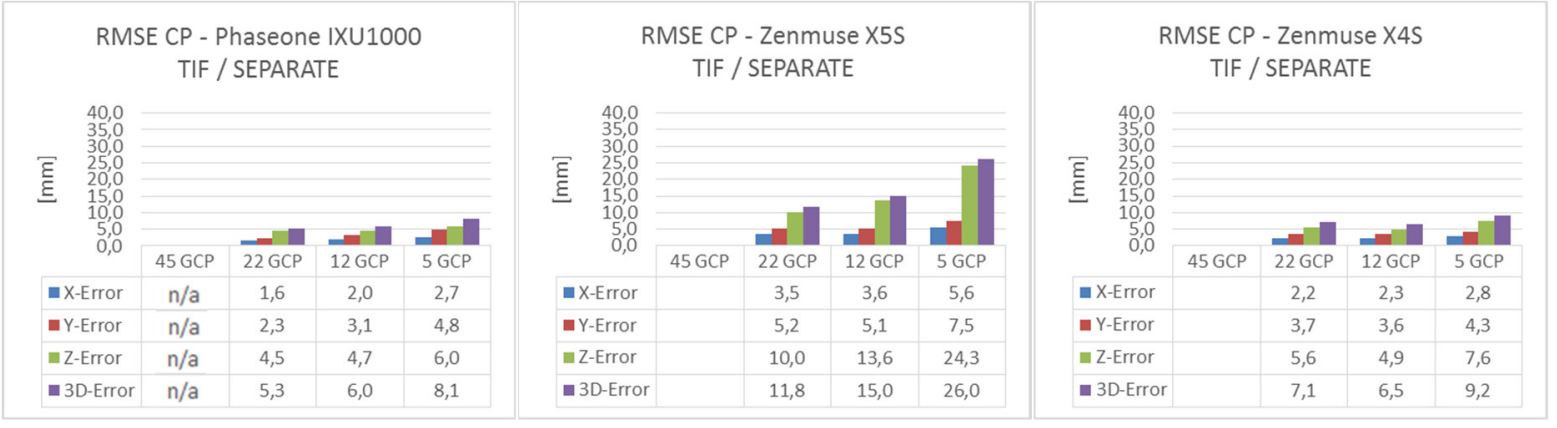

Figure 8. RMSE values at CPs. Flight arrangement: R+C. TWO sets of interior orientation parameters (SEPARATE). Calculation: Agisoft PhotoScan.

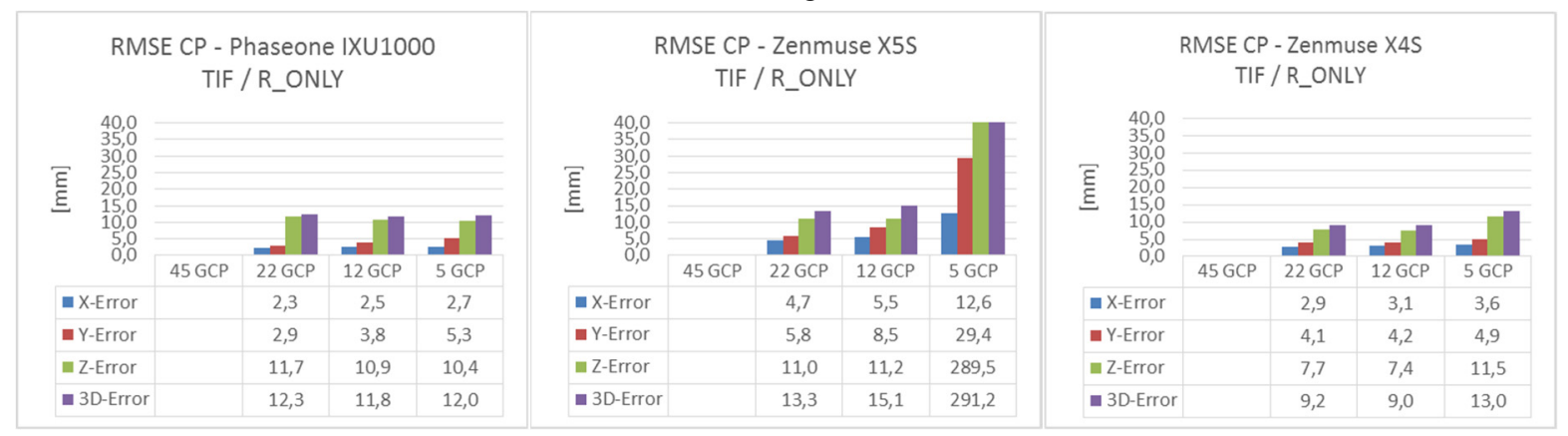

Figure 9. RMSE values at CPs. Flight arrangement: R. Calculation: Agisoft PhotoScan.

The Zenmuse X4S, the standard camera system of the Phantom 4 PRO (also usable with the Inspire 2) shows only slightly worse results. The 3D RMSE value is below the GSD, even with minimal ground control point distribution. The camera concept implemented in the Zenmuse X4S, comprising a fixed-focus lens and a mechanical central shutter, shows a high stability and resembles - at least in the context of the flights carried out here in a timely manner - almost metric characteristics. 
In contrast to the two other systems, the results for the Zenmuse $\mathrm{X} 5 \mathrm{~S}$ are significantly worse. The existing interchangeable lens in conjunction with the electronic focusing to infinity, ends up in a camera system that is not very stable in itself, with mechanical instability between lens and camera body at the bayonet. The RMSE values are 2-3 times worse than those of comparable systems and only lay below the GSD, when the control points are (typically: digital aerial cameras), however, this extended flight arrangement can be dispensed with a pre-calibration of the cameras.

The effects of a single flight (here: Regular $-\mathrm{R}$ ) on the examined UAV cameras appear to be very clear (Figure 9). While the mechanically more stable systems - Phase One and Zenmuse
RMSE GCP - (TIF) / UNIFIED

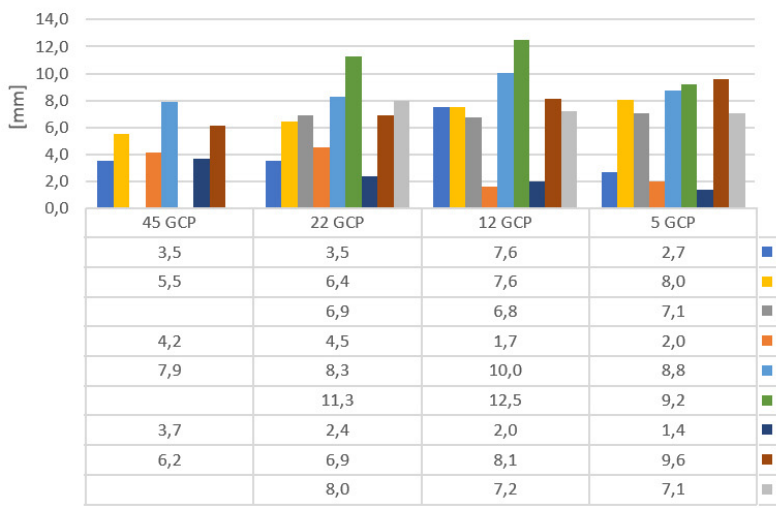

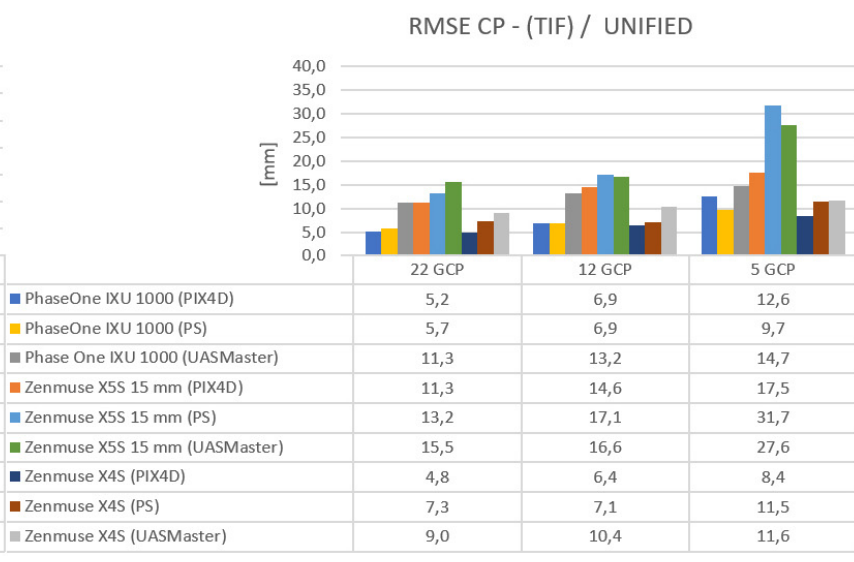

Figure 10. Comparison of the 3D RMSE values at GCPs (left) and CPs (right) as results of the bundle block adjustments with Pix4Dmapper, Agisoft PhotoScan and Inpho UASMaster. Note scaling.

close together (here $22 \mathrm{GCP}$ ).

\subsection{Effects of separate interior orientation parameters}

The main reason for carrying out cross flights via separate flights is based on the fact that commercially available flight planning tools (e. g. Map Pilot, Pix4DCapture) do not allow cross flights with varying flight altitudes. Besides that, RAW data storage in the DJI systems requires a reduction in flight speed to have sufficient time for storing images on the SD card (approx. a factor of 3 higher time requirement compared to the "DJI-JPG"). Storing images on the available and much faster SSD medium is not possible. It occurs that nobody - even DJI consultants knows why (information from late 2018)!

Consequently, a new setup must be carried out for the DJI systems before each flight, as these lose their settings temporarily after being without power due to the change of batteries.

This fact makes it reasonable to carry out the bundle block adjustment with separate interior orientation parameters for the partial flights within the scope of cross flights. The results are summarized in Figure 8. Although the results of the variants "RC UNIFIED" and "RC SEPARATE" are similar, it can be observed that the variant "RC SEPARATE" leads to increased accuracy, especially for the Zenmuse X5S. This tendency is a clear indication of the less stable interior orientation compared to the $\mathrm{X} 4 \mathrm{~S}$ and Phase One system.

Based on the reasons mentioned above (concerning the DJI system), it would make sense to be able to leave the cameras switched on, i.e. by using a buffer battery, upon changing of the power sources. In this case the setup would remain relatively stable at least for the connected missions.

\subsection{Effects of single flight arrangements}

A complete cross flight usually represents a considerable additional effort over the entire process chain, starting from the recording to the final evaluation. When using metric cameras
$\mathrm{X} 4 \mathrm{~S}$ - tend to display poorer results (in comparison to the crossflight configuration), the Zenmuse X5S shows significant loss in accuracy. Particularly affected is the height accuracy in conjunction with a reduced number of ground control points for the block referencing. The larger RMSE values (in Z-direction) result mainly from problems with the numerical determination of the parameter "focal length", for which the present R-block obviously offers minor depth information.

Beyond that, the positive effect of a very dense arrangement of control points is clearly visible in this block for the instable X5S. The results of the large-format Phase One are almost independent of the control point distribution, as can be seen from the RMSE values of the check points (CP). In principle, this observation also applies to the Zenmuse X4S.

\subsection{Comparison of the software products used}

The number of software products used to perform a bundle block adjustment (BBA) of UAV images is constantly increasing. Although it can be assumed that users of a certain company/organisation work with the same software, the photogrammetric community naturally uses different software applications in everyday life, which often do not lead to identical results.

Therefore, based on the Zollern test data sets, identical BBA using the Agisoft PhotoScan (Agisoft 2019), Pix4Dmapper (Pix4D 2019) and Inpho UASMaster (Trimble 2019) software were performed. All software systems are commercial products and have a considerable market presence.

The configuration of the created projects corresponds to the specifications for the respective software. As far as available, implemented "templates", with standard parameters, were used. The measurement of the signalized ground control points was carried out manually by the same operator, based on the measurement routines preinstalled in the programs. 
For this reason, the herein presented approach can be regarded as representative for project work. Thus, the results are largely unaffected by "extended expert knowledge".

Figure 10 shows the three-dimensional RMSE values after the bundle block adjustment, whereby a separate representation of position and height deviations is omitted here. The differences of the RMSE values are clearly visible for all examined cameras and the respective block references. In general, the calculations with Pix4Dmapper tend to result in significantly lower RMSE values than in the comparative projects which were carried out with PhotoScan or UASMaster. This applies to the ground control points (GCP) as well as to the check points (CP). Basically, it is difficult to find reasons for this, since technically the same methods are utilized. In contrast, different procedures can be assumed within the software, which, however, are less likely to be evaluated by users.

For the 22 and 12 GCP configurations all software products deliver a 3D RMSE below the GSD at CPs, except for the Zenmuse X5S which is worse, especially for UASMaster. Here the mentioned instability of the lens might play the major role. Interestingly for the 5 GCP configuration results vary considerably between the software products, indicating different strategies on how the residuals are distributed and the datum is defined.

The results shown in Figure 10 are of some relevance, as they represent one of the essential evaluation criteria for image orientation. Particularly in the context of engineering projects, which require an accuracy level of just a few millimeters, a quality assessment of the results achieved is rather difficult (e.g. concerning the question whether a defined accuracy has been reached).

\section{CONCLUSION AND OUTLOOK}

The in-situ tests of various UAV systems carried out on the basis of comparable data sets of the UAV test field at the Zollern colliery revealed remarkable differences in the performance of the cameras used. Surprisingly, the cheapest camera in the test, the Zenmuse X4S, yielded very good results.

Furthermore, the positive effects of cross flights (with differences in flight altitude of approx. 20\%) were confirmed. Unfortunately, the flight planning tools do not generally offer this variant as a standard concept, so that there should be a certain need for action on the supplier's side. This is accompanied by the necessity of self-calibration for the cameras used. None of the examined cameras can be described as a metric camera under consideration of the specific requirements coming from a high-precision UAV image flight. Taking into account the individual camera concept, it can therefore also make sense to introduce partial flights, with their own parameters of interior orientation, into the bundle block adjustment. The results also confirm conclusions from earlier studies, and of course the textbook knowledge, that the GCP distribution has a major effect on BBA quality. In this context, current developments of RTK-assisted GNSS positioning for rotary wing systems are very interesting and worth further evaluation. It needs to be validated whether similar effects are observable like confirmed already for fixed-wing systems (Gerke and Przybilla, 2016).

The final comparison of the calculated results using different software products, is in some way astonishing, as it shows clear differences in quality (accuracy) within the available data sets.

\section{ACKNOWLEDGEMENTS}

The author thanks all participants of the Zollern autumn campaign for their active support. In addition to the partners of Phase One and Coptersystems, special thanks goes to the students of the elective compulsory event "Engineering Surveying" 2017, who attend the study course Surveying at the HSBO (Head: Prof. Dr. Manfred Bäumker), for their motivated participation in the network measurement!

\section{REFERENCES}

Agisoft, 2019. Homepage Agisoft, https://www.agisoft.com, last access 17.01.2019.

$\begin{array}{llrl}\text { Coptersystems, } & 2019 . & \text { Multicopter } & \text { "CS8-MM", } \\ \text { https://coptersystems.com/produkte/cs8-mm/, } & \text { last access }\end{array}$ 17.01.2019.

Cramer, M. \& Krauß, H., 2008. Test field Vaihingen/Enz. www.ifp.uni-stuttgart.de/dgpf/pfg-2-08-anzeige.pdf, last access: 17.01.2019

Cramer, M.; Przybilla, H.-J., Meißner, H. \& Stebner, K., 2017. Kalibrierung und Qualitätsuntersuchungen UAV-basierter Kamerasysteme. Unmanned Aerial Vehicles 2017 (UAV 2017), Proceedings of the DVW, 86, 67-84, Wißner-Verlag, Augsburg.

Gerke, M. \& Przybilla, H.-J., 2016. Accuracy analysis of photogrammetric UAV image blocks: influence of onboard RTK-GNSS and cross flight patterns. Zeitschrift für Photogrammetrie, Fernerkundung und Geoinformation (PFG), 2016(1), 17-30.

Grayson, B., Penna, N. T., Mills, J. P., Grant, D. S., 2018. GPS Precise Point Positioning for UAV Photogrammetry. The Photogrammetric Record. Volume33, Issue164, pages 427-447

Kraft, T..; Geßner, M.; Meißner, H.; Przybilla, H.-J.; Gerke, M., 2016, Introduction of a photogrammetric camera system for UAV with highly accurate GNSS/INS information for standardized workflows. EuroCOW 2016, Lausanne, Switzerland, 10.-12.2.2016, (http://www.eurocow.org/)

Kraus, K., 1994, Photogrammetrie, Band 1. Grundlagen und Standardverfahren. Ferdinand Dümmlers Verlag, Bonn, 263 S.

Lindstaedt, M. \& Kersten, T., 2018, Zur Bedeutung von Passpunkten bei der Aerotriangulation UAV-basierter Bildflüge. UAV 2018 - Vermessung mit unbemannten Flugsystemen, Proceedings of the DVW, 89, 81-101, Wißner-Verlag, Augsburg.

LWL, 2019, Website of industrial museum Zollern colliery, https://www.lwl.org/industriemuseum/standorte/zeche-zollern, last access: 17.01.2019

Mueller, C. \& Neumann, K., 2016, LEICA DMC III Calibration and Geometric Sensor Accuracy. The International Archives of the Photogrammetry, Remote Sensing and Spatial Information Sciences, 40(3/W4), 1-9.

Nex, F., Gerke, M., Remondino, F., Przybilla H.-J., Bäumker, M., Zurhorst, A., 2015. ISPRS Benchmark for Multi-Platform Photogrammetry. ISPRS Annals of the Photogrammetry, Remote Sensing and Spatial Information Sciences, Vol. II-3/W4, pp.135142. 
Oniga, V.-E., Pfeifer, N., Laghin, A.-M., 2018, 3D Calibration Test-Field for Digital Cameras Mounted on Unmanned Aerial Systems (UAS). Remote Sensing 2018, 10(12), 2017; doi: $10.3390 / \mathrm{rs} 10122017$

Phase One, (2019). Website https://industrial.phaseone.com/ Mapping_Surveying_Applications.aspx, last access 17.01.2019. Pix4D, 2019, Website https://www.pix4d.com/, last access 17.01.2019.

Przybilla, H.-J., Reuber, C., Bäumker, M. \& Gerke, M., 2015. Untersuchungen zur Genauigkeitssteigerung von UAVBildflügen. Proceedings of the German Society of Photogrammetry, Remote Sensing and Geoinformation (DGPF), 35. Annual scientific-technical conference of DGPF, 24, 45-54.

Przybilla, H.-J., 2017. Kameras für UAS - Eine Marktübersicht. In: Unmanned Aerial Vehicles 2017 (UAV 2017), Proceedings of DVW, 86, 51-66, Wißner-Verlag, Augsburg.

Przybilla, H.-J., Bäumker, M. \& Vieten, J., 2018. Das UAVTestfeld Zeche Zollern in Dortmund. UAV 2018 - Vermessung mit unbemannten Flugsystemen, Proceedings of the DVW, 89, 61-80, Wißner-Verlag, Augsburg.

Trimble, 2019. Website https://www.trimble.com/ geospatial/inpho-uasmaster, last access 28.03.2019

Wikipedia, 2019. Industrial museum Zollern colliery, DortmundBövinghausen. https://de.wikipedia.org/wiki/Zeche Zollern. last access: 17.01.2019 